\title{
NOTE ON THE STERILISATION OF HANDS.
}

\author{
Bx W. S. HAUGHTON, M.D.; B.CH., \&c., \\ Surgeon to Dr. Steevens' Hospital;
}

AND

ROBERT J. ROWLETTE, M.D. DuBL., Pathologist to Dr. Steevens' Hospital, and to the Rotunda Hospital;

Lecturer in Pathology, Queen's College, Galway.

[Read in the Section of Surgery, December 1, 1905].

A YEAR ago $I$ had the honour of bringing under the notice of this Section some remarks on the value of aseptic technique, in the course of which I referred to the "sterilisation of skin" as the weakest link in the chain of asepsis. Reference was made to a series of investigations on this point which I have been carrying out during the past few years, and my communication to-night with my colleague, Dr. Rowlette, is a further contribution to the subject.

During the last few years much attention has been directed, at home and abroad, to skin disinfection, by many workers-amongst whom I would mention Kocher, Mickulicz, Ahlfeld, Fürbringer, and Leedham Greenand the general consensus of opinion seems to be that while there are several good methods the best is the "soaphot-water-alcohol" method. My previous experiments confirm this view, but as my own skin shows some intolerance to alcohol, during the past twelve months I have devoted attention to the "soap-hot-water-sublimate" method in aqueous solution. My object in so doing was to test thoroughly the efficiency of one single method when applied to a number of different pairs of hands, under the varying conditions of one's assistants, residents, and 
nurses, rather than to try a number of different methods, the results of which would necessarily be of less value from the more limited application.

The results of this method Dr. Rowlette will tell you from the bacteriological standpoint.

Clinically speaking, I am satisfied the method is good, and, in my own experience, equally good with the "soaphot-water-alcohol" method, as tested by:-

(1) Primary adhesion of wounds.

(2) The small amount of resulting scar tissue.

(3) Absence of pain or other signs of inflammation.

(4) Absence of the train of symptoms we call "shock" (surgical).

(5) The rapid return of a healthy appetite, even after major operations, which I generally gratify fully on the third day.

Of course different methods may yield equally good results, as exemplified in the practice of different workers, and $I$ am prepared to admit that special methods may suit individual operators, which leads us to consider the "personal factor" in individual skins. But it is our duty as surgeons to seek for the method which, with the widest application, yields the most consistent and best results. And it is further our duty to have the courage of our convictions, and frequently subject our own methods to the searching light of bacteriological proof.

The "personal factor" in skins is, in my opinion, not sufficiently regarded, and includes such considerations as :- -

(1) Abstention from touching septic surfaces, except through the medium of rubber gloves.

(2) The appreciation of the meaning of cleanliness, and the mechanical capacity of scrubbing every part as clean as possible. 
(3) The personal health and texture of a particular skin, which varies enormously in different individuals. Some skins being able apparently to keep themselves comparatively sterile, while others harbour bacteria at all times. This, I believe, accounts for the good results sometimes obtained by methods of preparation which appear insufficient and even careless.

(4) The rate of sweating (both sensible and insensible perspiration). Skins which remain dry under the trying strain of heated and moistened theatre air, to say nothing of the mental strain of anxiety, have an enormous advantage as regards duration of comparative sterility.

(5) The nature of skin surface. If this be soft and smooth, which, of course, betokens health, and implies a healthy reaction to bacterial invasion, it is much easier to disinfect. The use of any antiseptic or chemical poison is liable to produce dermatitis; and no matter how healthy a skin may be, if many operations have to be performed, especially daily operations, it is only a question of time and frequency when the skin must give way, more or less, under the strain of a summation of stimuli.

It is to such skins especially that aseptic technique and rubber gloves are in the highest degree advantageous.

Our method of preparing the hands is:-

(1) Scrubbing, from fifteen to twenty minutes by clock, in very hot water.with soap and nail brush. A battery of nail brushes is boiled for twenty minutes, immediately before and after use, and stored in the interval of using in a boiled tray containing 1-500 sublimate solution. The scrubbing must be very thorough and painstaking, visiting every particle of skin of fingers, nails, hands and forearms. seriatum several times. There is a very considerable. difference in the "personal equation" of each individual in carrying out this, and most of the success of the method 
depends on thoroughness. A stream of running water is preferable to a basin, and if the latter must be used, several changes of water are desirable.

(2) Then the hands are plunged into and rubbed with a solution of 1-500 sublimate in a boiled bowl for one to two minutes.

(3) Next they are rinsed in a boiled bowl of sterilised normal saline to free them from sublimate.

(4) They are put into long rubber gloves, which have been boiled for five to ten minutes, and placed in a boiled bowl of sterile normal saline.

(5) The gloved hands are then steeped in sublimate 1-500 for four to five minutes, which is rinsed off just before commencing operation in another bowl of sterile saline. This is done to preserve the wound from the entrance into it of any chemical poison.

Air-borne infection of the gloves during operation is met by washing gloves every fifteen to twenty minutes in sublimate 1-500, which is again rinsed off in normal salinetwo bowls containing these solutions being placed on tables at the operator's and assistants' elbows for frequent and ready use.

Dr. Rowlette will describe to you the results of our tests carried out at various stages of this preparation. As I have already stated, the clinical results of my practice of this method are exceedingly satisfactory.

The prolonged scrubbing removes a considerable amount of dirt and epidermis containing bacteria, and apparently arrives at a layer of skin less highly charged with bacteria. The sublimate disinfects and somewhat hardens this layer, and the rubber gloves in our experiments maintain an unbroken aseptic surface throughout the longest operation.

The results of our experiments confirm the generally accepted view that sterilisation of the skin is difficult or 
impossible, and when obtained is not permanent, which is the strongest possible argument for the use of rubber gloves.

In considering the various methods of testing sterilisation of skin hitherto adopted we came to the conclusion that they did not satisfy us, because they were not suffciently stringent. They did not satisfy what may be termed "service conditions" - that is, the condition of an ordinary operation. The superficial area of skin examined was too small, the nails were not searched deeply enough, or the conditions were artificial.

We, therefore, adopted a method, which Dr. Rowlette will describe, in which all ten fingers are examined, back and front, besides back and front of hands and wrists, and all ten nails are examined deeply.

If, therefore, our experiments show a relatively high percentage of positive results in bacterial growths, it must be borne in mind that our test is one of the most stringent yet put forward, and that in estimating results we aim at an absolute standard of sterility and not a comparative one in which a certain arbitrary number of bacterial colonies are considered as a practical proof of sterility. These facts, we believe, speak for themselves.

By Dr. Rowlette.

Is deciding on the tests by which to estimate the efficiency of the method of disinfection which has been described, our endeavour.has been, on the one hand, to employ such tests as would approximate, in their severity, to the conditions met with in the course of surgical operation, and, on the other, to avoid any test which might be considered unduly artificial. We have thought it important, more- 
over, to subject the hands of the assistant and of the aseptic theatre sister to the same tests as we applied to the hands of the surgeon, both on account of the practical importance of controlling every source of infection, and because we were thereby able to test the method on a larger number of different skins.

The actual method adopted has been as follows:-At the end of fifteen to twenty minutes' washing in soap and water, swabs sterilised by boiling in water for at least ten minutes, and then squeezed by the hand to be tested, have been "rubbed along the entire length of the hand and fingers, front and back, of each hand, covering a surface of about 80 square inches, and the swab then smeared over the surface of sterilised agar in a culture tube. This was repeated on the three pairs of hands. A stout platinum wire, sterilised by heat, was then dragged under each of the ten nails, the skin being drawn away from the nail, and a culture tube of agar inoculated therewith. When the hands had been subjected to the sublimate lotion, and well rinsed in saline solution, the same process was repeated.

As soon as the gloves had been drawn on, their outer surfaces were rubbed with swabs in the same way. This served both as a test of the sterility of the gloves and as a control on the other experiments. At the end of operation, with the gloves in actual working condition, cultures were similarly made. When the gloves were removed the hands were again tested as before by swab-cultures. These tests seem to us as severe as any used by other investigators, and much more severe than those used by most, while at the same time they produce conditions similar to those to which the hands are subjected in actual operative work. In this manner close on two hundred observations were made, extending over a period of six months, and 
covering several changes in the assistant, and some changes in the sister employed.

We give here percentages of the results obtained at each stage of the proceedings, summing together those from the three pairs of hands:--

After washing in soap and water alone growths from swabs occurred in 56.6 per cent.; and from nails in 79.3 per cent. After the use of sublimate lotion growths from swab-cultures from the hands appeared in 13.3 per cent.; and from nails in 66.6 per cent. From the gloves when first put on growths occurred in not a single case. From the gloves at the end of operation, growths occurred in one ease only. Swab-cultures from skin of the hands at the end of operation gave growths in 62.5 per cent.

The operations occupied very varying lengths of timethe shortest taking 35 minutes, and the longest 1 hour 55 minutes.

In most cases only one organism was found in the growth, though in a few tubes two different organisms occurred. The following were identified, as far as cultural and morphological characteristics go:-St. p. albus in 68 per cent. of all growths; st. p. aureus, 11 per cent.; st. citreus, 5 per cent. The other organisms found included a diplococcus resembling diplococcus pneumoniæ in 3 per cent., and various common non-pathogenic air organisms. The cultures were incubated for at least seven days before final examination.

In studying these results one is first struck by the fact that whereas the use of sublimate solution apparently reduced the infectivity of the surfaces of the hands considerably (56.6 to 13.3 per cent.), it only reduced that of the nails slightly (79.3 to 66.6 per cent.). This, I think, may be explained by the fact that dirt embedded under the nails is very difficult to reach by any antiseptic fluid 
or mechanical process of cleansing. The antiseptic probably coagulates the superficial layers of dirt, leaving the under layers unaffected.

Moreover, it is to be remembered that it is difficult to be sure that the sublimate is entirely rinsed off the hands by the saline solution. If any remain, some of it may be absorbed by the swab, and the growth of organisms present may be inhibited. The fact that in one case only was any germ found on the gloves at the end of operation shows how effective a protection sterilised gloves constitute. It is to be noted, too, that in many cases gloves, apparently intact, were found afterwards to contain a few minute punctures, so that the swab taken at the end of operation must have absorbed at least a little fluid from inside the gloves. It will be noticed that the infectivity of the hands (62.5 per cent.) on removal of the gloves was slightly above that after the simple washing in soap and water (56.6 per cent.), and considerably above that of the hands immediately after the use of antiseptics. We are of opinion, however, that the examination of the skin of the hands by swabs, or indeed by any of the methods devised, while the epidermis is possibly sodden with antiseptic fluid, is fallacious. The increase in the infectivity of the surface of the skin during operation is, of course, due to organisms working their way, by means of sweat or otherwise, from the deeper layers to the surface.

Various minor points of interest were noted during the conduct of these experiments. Thus, any marked unevenness or irregularity of the skin in any of the subjects examined meant that disinfection was more difficult. During the early part of the series one of the subjects suffered from a wart on the palmar surface of a finger, and while this persisted swabs almost invariably gave positive results. On one occasion the period of washing in 
water was shortened to 5 to 10 minutes, with a markedly increased percentage of positive results.

It is not the purpose of this note to discuss the subject of sterilisation of the hands as a whole, but it is necessary to point out the bearing of our experiments on current opinions. It seems to be now generally admitted that absolute sterilisation of the hands is impossible, and we find rarious investigators laying down arbitrary standards of the maximum number of colonies which they consider to fall within the limit of "practical sterility." The futility of any such standard need hardly be pointed out, depending as it does on individual methods of testing impossible to standardise, and on an entirely arbitrary numerical limit. Our aim, on the other hand, has been in each case to decide whether absolute sterility has been attained, as, if not, there is no guarantee against infection. Our experiments, as far as one method of disinfection goes, show that sterility is unattainable in more than half the cases, and that even where the surface is at first apparently sterile, prolonged sweating brings to the surface a large number of bacteria. This same fact has been proved with great care by Dr. Rupert Collins, who showed the enormous increase in the infectivity of hands when subjected to prolonged sweating produced by the injection of pilocarpine.

We think, then, that we are justified in concluding:-

(1) That washing in soap and water for 15 to 20 minutes, and in sublimate lotion 1-1000 for two minutes, does not guarantee sterility of the hands.

(2) That during the course of an operation bacteria previously buried are carried to the surface.

(3) That rubber gloves offer a sufficient and practically complete protection. 